\title{
Disjoint connectivity of wireless sensor network
}

AKADÉMIAI KIADÓ

\section{International Review of Applied Sciences and Engineering}

$11(2020) 2,107-114$

DO1:

$10.1556 / 1848.2020 .20005$

(c) 2020 The Authors

\section{ORIGINAL RESEARCH PAPER}

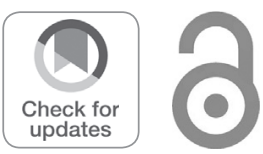

*Corresponding author. E-mail: alaaaltimimy@ uomustansiriyah.edu.iq

\author{
AHMED S. AL-ZUHAIRI, ALAA H. ALTIMIMY*, ALI T. AL-AQBI \\ and HAZIM M. AL-KARGOLE
}

Computer Engineering Department, Mustansiriyah University, Baghdad, Iraq

Received: June 10, 2019 • Accepted: November 10, 2019

Published online: July 28, 2020

\begin{abstract}
Growing ramification in WSN contemplations are not restricted to routing, construction of protocols, dynamic of mobile nodes and infrastructure of the network. Although transcended to geometric level demonstrated as computational and dynamical geometries in spite of graph theory. In this paper we present step forward recognition features of a network devoted to solve the problem of reconstructing the disconnected network by connecting any disconnected chains. It considers geometrical properties of random depletion nodes deviated from unit grid. Number of chains and number of nodes in each chain are calculated with the average number of connections to a total nodes and longest chain. Histogram represented number of chains and numbers of nodes for each chain are used to show fragmentation of the network. Algorithm included a method to translate adjacent matrix to chain matrix and vice versa to check the agreement of initial case with the results. The amount of chain deviation and average connections per node for longest connected chain and for the total network are drawn as bar charts and conduct interpretations.
\end{abstract}

\section{KEYWORDS}

networks, WSN, disjoint connectivity, matLab, random depletion

\section{INTRODUCTION}

WSN is one of active and operational subjects, although the extended care to apply it $[1,2]$, many levels of studies are considered like infrastructure technology, electronics, microprocessors, digital signal processors DSP, communications, battery production technology and others of power sources [3-6]. Additional subjects are involved as network management, routing, types of protocols applied. Estimating capacity of transmitted data, network lifetime, maintenance, and fault tolerance are topics of important role in studding and implementing WSN [7-9].

Infrastructure of network includes methods of how the node distributed in the range of interest (RoI) and sometimes accesses the exact coordinates of each node (two or three dimensions). Knowing exact coordinates of nodes plays an important role especially in applications like indicating fire in a forest or a leakage in a petroleum pipe. The mechanism of allocated node position called localization while applying statistical information about node position, the number of connections between nodes may give approximate information about the efficiency and capacity of the network. The way of depletion nodes across RoI may play an important factor to all performants of the network and affects connectivity, coverage, routing and traffic of packets as the capacity of the node system.

WSN from geometric point of view is important like other studies, almost all scientists or engineers working on WSN do not give geometry of network any consideration according to replace the whole routing problem to topology and tree connection between nodes or depend on statistical information and leave geometry as a losing end especially when position of node is relevant to the application [10-12]. Although geometry of a network is connected to a graph theory and computational geometry, these branches of mathematics lead to dynamic geometry that will bring network geometry to new fields and interact with more general aspects and concepts in both ways $[13,14]$. Beginning with localization is a keystone to solve 
problems of network. Ubiquitous software is applied for simulation of WSN, like opnet, $\mathrm{C}++$ and MATLAB programs, they are working over different levels of obligations and complexity. Estimating packet transfer capacity acting with different structures of protocols and verity of node connection methods are one level. Simulating the details of electronic block diagram circuit defined each WSN node, reading communication patterns and capability of transmission, then time response and synchronization of all that are another level $[15,16]$.

Prim topics in WSN are connectivity and coverage. Connectivity is about determining the connection between nodes - homogeneous or heterogeneous - and finding losing end chains or isolated nodes, although calculating area coverage is a complementary topic to connectivity [6, 17] and a way to notice the efficiency of WSN to handle, calculating area coverage need to discriminate chains and isolated nodes, which is the topic of this paper, then find area coverage for each.

In this paper we encounter estimating number of chains (connected nodes but disjoint from other nodes), number of nodes in each chain and average connections to a longest one and compare that with the average connections to all nodes in the network, this is done with different number of nodes, different densities and verity of maximum transmission distances. Within many years of research, we did not find any independent attempt to find geometric properties of WSN. Studding included chains in the network is an algorithm used to predict efficient routing; this algorithm recognizes many chains in the network with predefined number of nodes, then selects suitable node within each chain and discriminates it as prime node. It is used as a connected node to main node or connected to a sink node. This algorithm simplifies routing techniques for the network $[18,19]$. Defined number of chains and the length of each chain gives important parameters to evaluate the properties of network and it is a step to word find algorithms to connect these chains in a proper way, also estimating average connection of nodes in chain and in a total graph indicate side of the properties of the network [20]. Detecting many chains in the network and the longest chain length is small fraction of the total nodes (diversity is about one) this network is of poor efficiency and cannot be used to any communication tasks, while if a diversity near zero indicates that more nodes are connected to main chain then transmission rate will be better. If network is disconnected a suitable technique may be used like network control, increasing transmission radius, or repositioning mobile nodes to establish connections to fragmented or insulated parts, the two methods are actually used [21].

\section{FIND THE LONGEST CHAIN ALGORITHM}

\subsection{Algorithm attended}

To find the longest chain in connected graph of simulated wireless sensor network algorithm that is written to simulate random depletion of nodes and find connected graph within maximum transmission radius then new method is written to find connected chains of nodes and estimate the longest one, and the algorithm is used to find statistical results for connected nodes in random distributed WSN.

The details of algorithm are explained in the following steps:

Step1: Estimate arbitrary coordinate matrix XY0:

(a) Set uniform grid $x \& y$ coordinates of a predefined grid step.

(b) Add random normal distribution of $x \& y$ coordinates of zero mean value and variation of 1 or 2 grid step.

Step2: Find connected matrix by calculating distance between nodes and connect $\left(a_{\mathrm{ij}}=1\right)$ them if distance $\left(d_{\mathrm{ij}}\right)<$ maximum transmission radius $\left(R_{0}\right)$ to generate connected matrix [22].

Step3: Find connected chain matrix by constructing a sequence of connected nodes to current node, these groups of nodes may be connected to each other and construct a chain Part of Mat-LAB $\mathrm{m}$-file to generate chain matrix from connected [23].

Step4: Find optimization connected group matrix by checking each sequence with the previous, if any common node combining the two sequences without repeated nodes.

Step5: Change maximum transmission radius $\left(R_{0}\right)$ and repeat from step2.

Step6: Draw longest chain on original connected graph as shown in Fig. 1.

Step7: Create histogram in Mat-LAB m-file if number of chains has the same number of nodes and draw it for each maximum transmission radius.

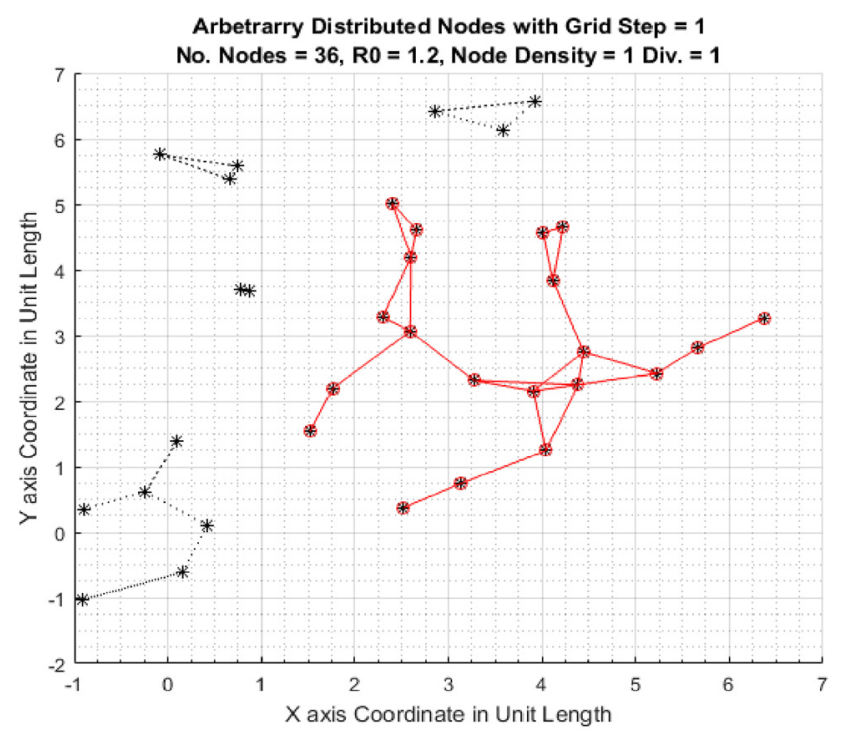

Fig. 1. Sample of Plot of original connected graph and longest chain marks with different color 
Step8: Calculate:

diversity factor $=1-\frac{\text { no. nodes in longest chain }}{\text { no. of node in total graph }}$

Average connections in graph $=\frac{\text { no. connections in network }}{\text { no. of nodes in total network }}$

Average con.s in longest chain $=\frac{\text { no. con.s in longest chain }}{\text { no. of nodes in longest chain }}$

Step9: Using Microsoft Excel program to collect data of diversity factor and average connections for total network and longest chain and draw bar charts for collected data for each collected case with different maximum transmission radius and deviations.

\subsection{Applied algorithm}

Algorithm created above is achieved using MatLab m-files and executed with:

1. Uniform grids $5 \times 5$ or $10 \times 10$ units with step value 1 or 2 .

2. Deviation of $0.5,1$, and 2 units.

3. Density of 1,4 , and 9 node/unit squared.

4. Radius of $0.95,1.2,1.5,1.95$, and 2.5 for density 1 node/ unit squared,radius of $0.475,0.6,0.75,0.975$ and 1.25 for density 4 nodes/unit squared,and radius of $0.13667,0.4$, $0.5,0.65$ and 0.8333 for density 9 nodes/unit squared.

Application success of algorithm includes two stages:

1. Number node connected matrix is produced from adjacent matrix making chain connections according to the applied algorithm, then adjacent matrix is reproduced from new matrix, comparisons are achieved by drawing

Table 1. Maximum transmission radii are compared for different depletion densities

\begin{tabular}{cllllll}
\hline Density (nodes/Unit Squared) & $R_{\max 1}$ & $R_{\max 2}$ & $R_{\max 3}$ & $R_{\max 4}$ & $R_{\max 5}$ & Remarks \\
\hline 1 & 0.95 & 1.2 & 1.5 & 1.95 & 2.5 & $R_{\operatorname{maxi}}$ \\
4 & 0.475 & 0.6 & 0.75 & 0.975 & 1.25 & $R_{\operatorname{maxi}} / \sqrt{4}$ \\
9 & 0.35 & 0.4 & 0.5 & 0.65 & 0.733 & $R_{\operatorname{maxi}} / \sqrt{9}$ \\
\hline
\end{tabular}

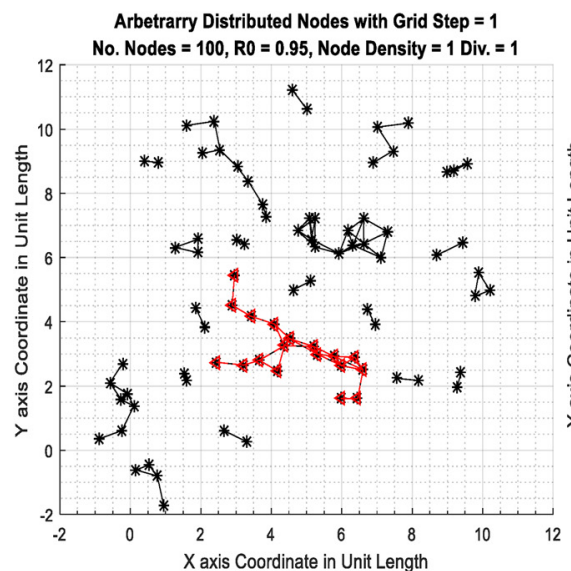

(a)

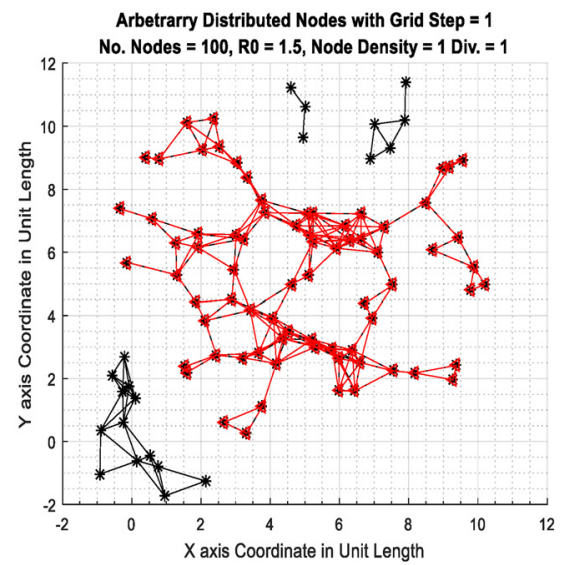

(c)

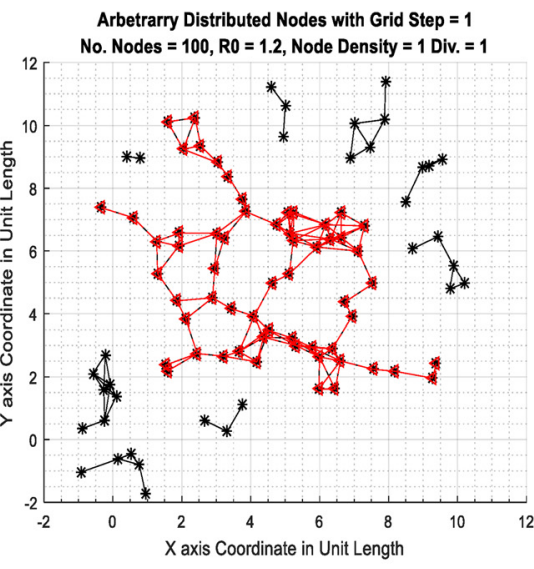

(b)

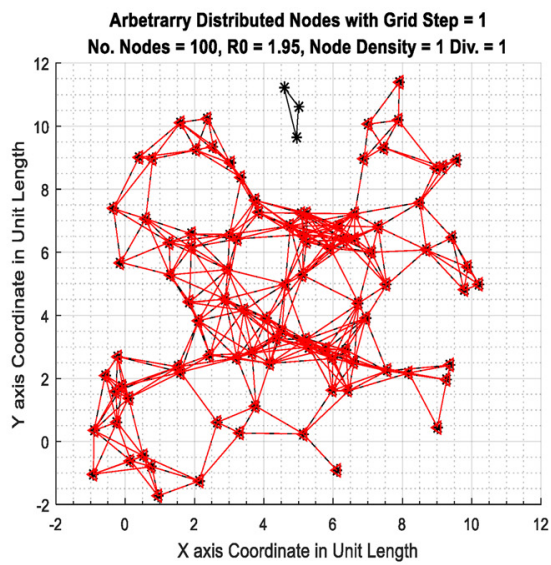

(d)

Fig. 2. Connected graph and the longest chain obtained with $R_{0}=$ (a) 0.95 (b) 1.2 (c) 1.5 and (d) 1.95 units 


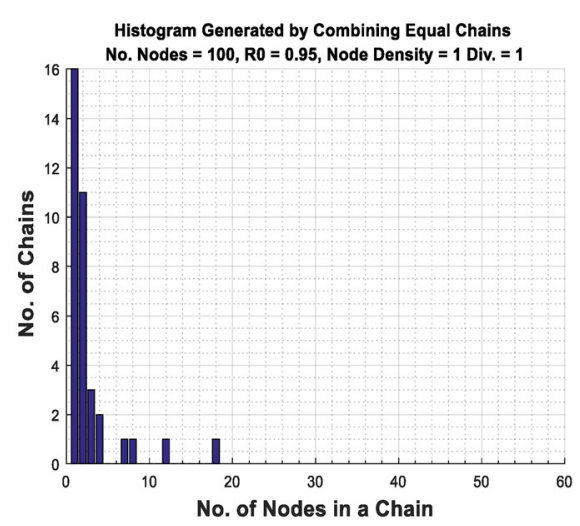

(a)

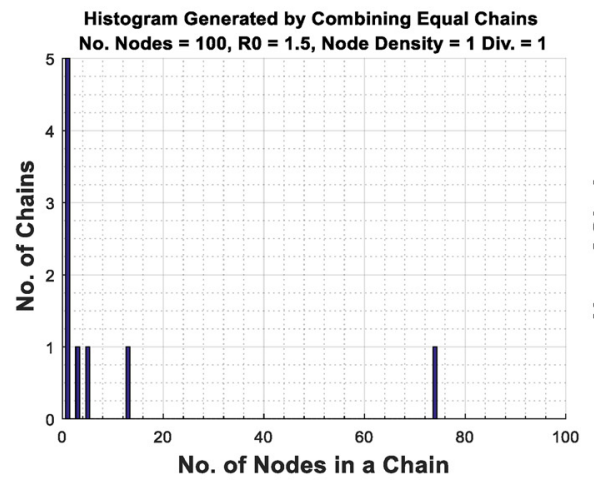

(c)

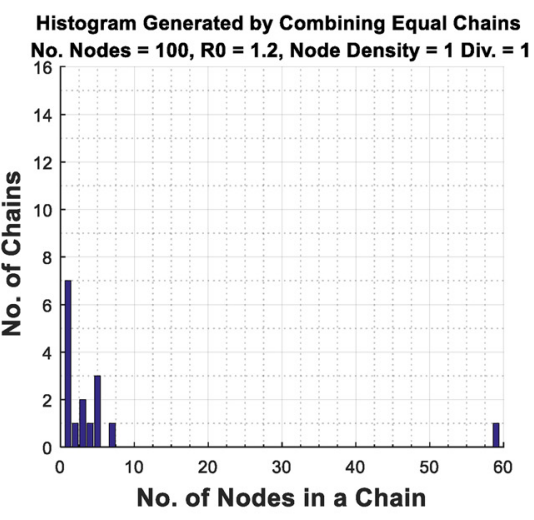

(b)

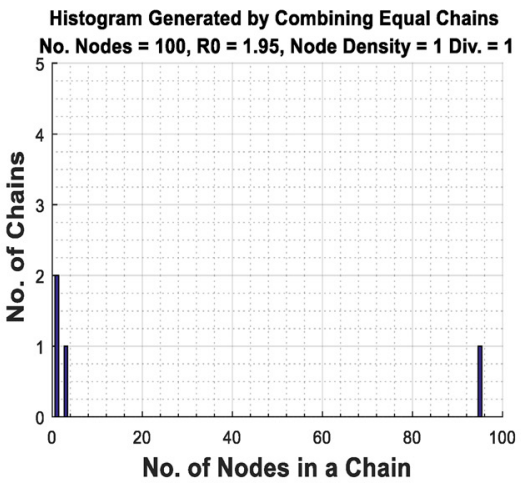

(d)

Fig. 3. Histogram of number of chains have any number of connected nodes for 100 nodes with node density 1 node/unit squared and unit diversity factor for different maximum transmission distances $R_{0}$ (a) 0.95 (b) 1.2 (c) 1.5 \& (d) 1.95 units

old and new adjacent matrices connected graphs, both graphs are matched. This test helps to accurate algorithm mechanism from bugs and miscalculations.

2. Longest chain in network is redrawn on original connected graph, so match of the longest chain is ensured directly on graph. Therefore, redrawing new adjacent matrix is no longer important since no new information can be noticed any more.

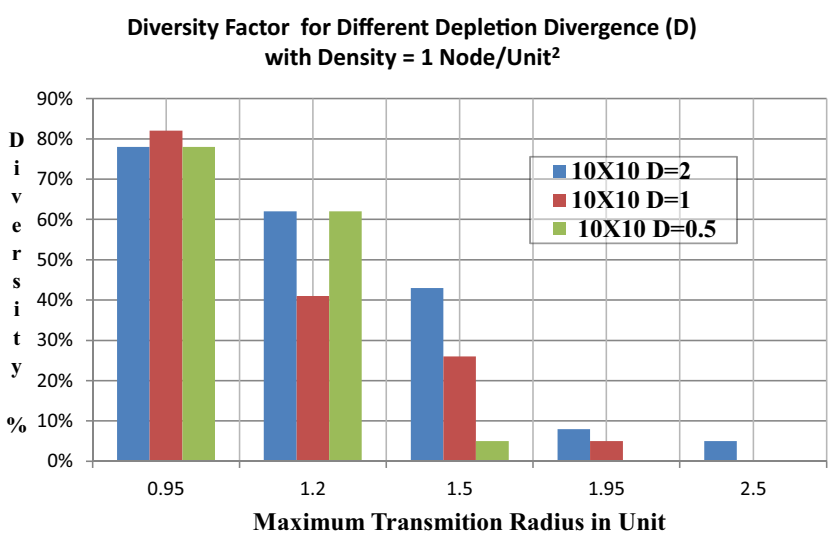

Fig. 4. Diversity factor with deferent deviations and maximum transmission radius $R_{0}$
To ensure accuracy of the algorithm small network is used as examples to check results with manual calculations and estimations, like number of nodes in a longest chain, number of chains formed, and number of connections per chain. All examples fit the results.

Many equations are used to define magnitude of chain deviations:

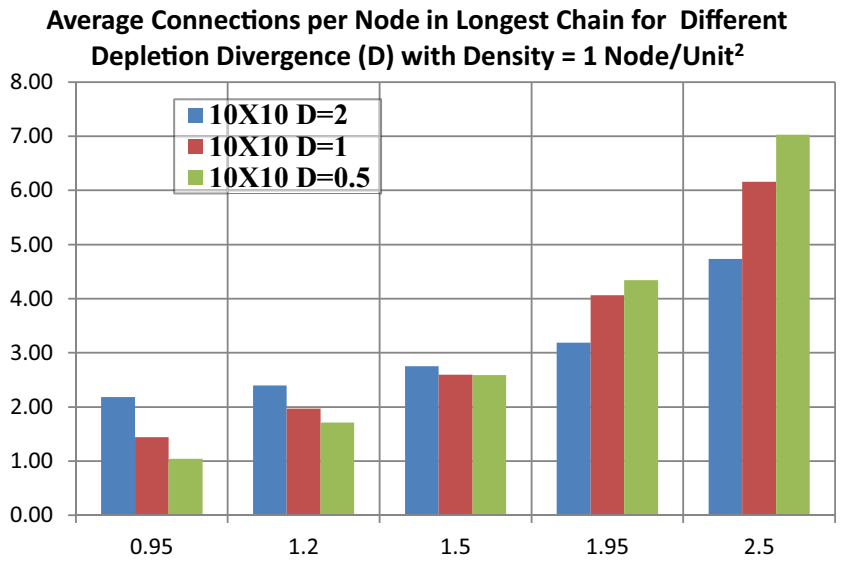

Fig. 5. Average connection per node in longest chain with deferent $D$ and $R_{0}$ 
Diversity Factor $=1-\frac{\text { No. of Nodes in Longest Chain }}{\text { No. of Nodes in Total Graph }}$

Avarage Connections in Logest Chain

$$
=\frac{\text { No. Connections in Longest Chain }}{\text { No. Nodes in Longest Chain }}
$$

No. Nodes in Longest Chain for Different Depletion Divergence (D) with Density = 1 Node/Unit ${ }^{2}$

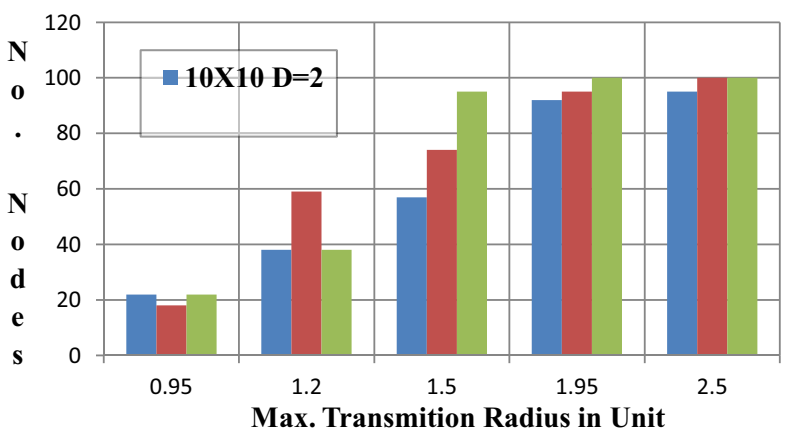

Fig. 6. No. of node in longest chain for deferent $D$ and $R_{0}$
Avarage Connections in Total Graph

$$
=\frac{\text { No. Connections in Total Graph }}{\text { No. Nodes in Total Graph }}
$$

\section{THE RESULTS OF FINDING LONGEST CHAIN ALGORITHM}

First: The average connections of nodes in longest chain are always more (or equal only when all nodes in network are connected to longest chain) than average connections of the network, see Table 1. This is self-evident, since in network many nodes are single or with few connections, contrary to connected chain.

Second: Deviations of chains in a network is big when maximum transmission radius is less than grid step. It seems that a dramatic change will occur when radius of maximum transmission is greater than three fourth of the grid step, $\mathrm{R}_{0}>0.75^{*} \mathrm{DG}$ as shown in Fig. $2 \mathrm{a}, \mathrm{b}, \mathrm{c}$, and $\mathrm{d}$.

Third: Histograms of the four cases previously mentioned are drawn, these histograms emphasize clearly
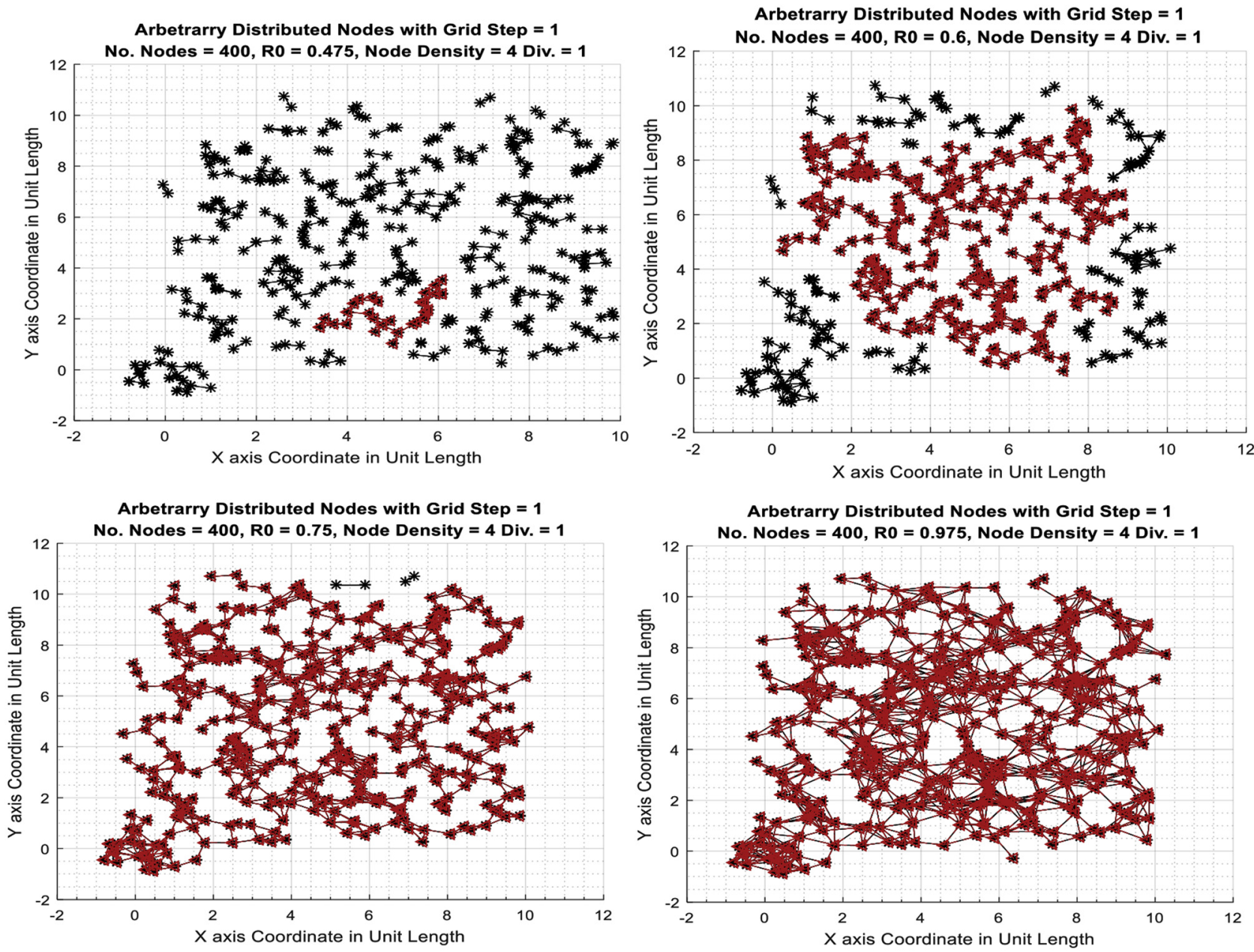

Fig. 7. Connected graph for $10 \times 10,4$ nodes/unit squared with $R_{0}=(a) 0.457$ (b) 0.6 (c) 0.75 (d) 0.975 units 
what graph plots of connected nodes show. Many different node number short chains occurr when maximum transmission radius is small compared to grid step and many single nodes also as shown in Fig. $3 a$ and b. While at maximum transmission radius greater than three fourth of grid step a clear long chain of nodes occurs, as shown in Fig. $3 c$ and $d$.

Forth: Diversity factor (the percentage ratio of number of connected nodes in longest chain to the total number of overall nodes in area) and average number of connections per node for longest chain and overall nodes are estimated according to Eqs (1) and (2) with respect to different maximum transmission radius. Bar chart created by Excel table shows that a large disperse of chains occurs when small maximum transmission radius is used. While, when radius of transmission being larger than three fourth of grid step disperses will be rapidly decreases and connections of longest chain may cover all nodes especially when maximum transmission radius approaches full step of grid. When the magnitude of deviation about grid crosses tends to change the behavior of connections are the same for different diversities with small differences. In large deviation more nodes on edges of the area may be deviated far from age and not connected to their neighbors, as shown in the first series in Fig. 4 for deviation of two units, although maximum transition radius is large, deviation still has effected the connections.

Any longer transmission radius will case more and more power dissipation and shorter battery and network life. Also, there will be more and more average connections to longest chain in the network, so the average of number of connections of longest chain also depends on measuring the quality of services (QoS) of the network. Average number of connections per connected node in a longest chain is recorded and drawn as an Excel bar chart. Figure 5 shows that when depletion deviation is equal to one unit, results will be medium compared with smaller or larger deviations. Also connections will be greater if the deviations of nodes are smaller when maximum transmission radius is at three fourth of grid step, that is local factors act between nodes, while when maximum transmission radius is bigger, this case will disappear, and adjacent nodes of different grid crossing have the domination effects as shown in Fig. 6.

Fifth: For dense depletion nodes a smaller maximum transmission radius is needed. Since connections between nodes depend on the mean distances between them, the expectation maximum transmission radius will be reduced as a square root of a uniform increasing in depletion density especially the area under consideration is squared. So to keep comparisons between radii of different density depletions it
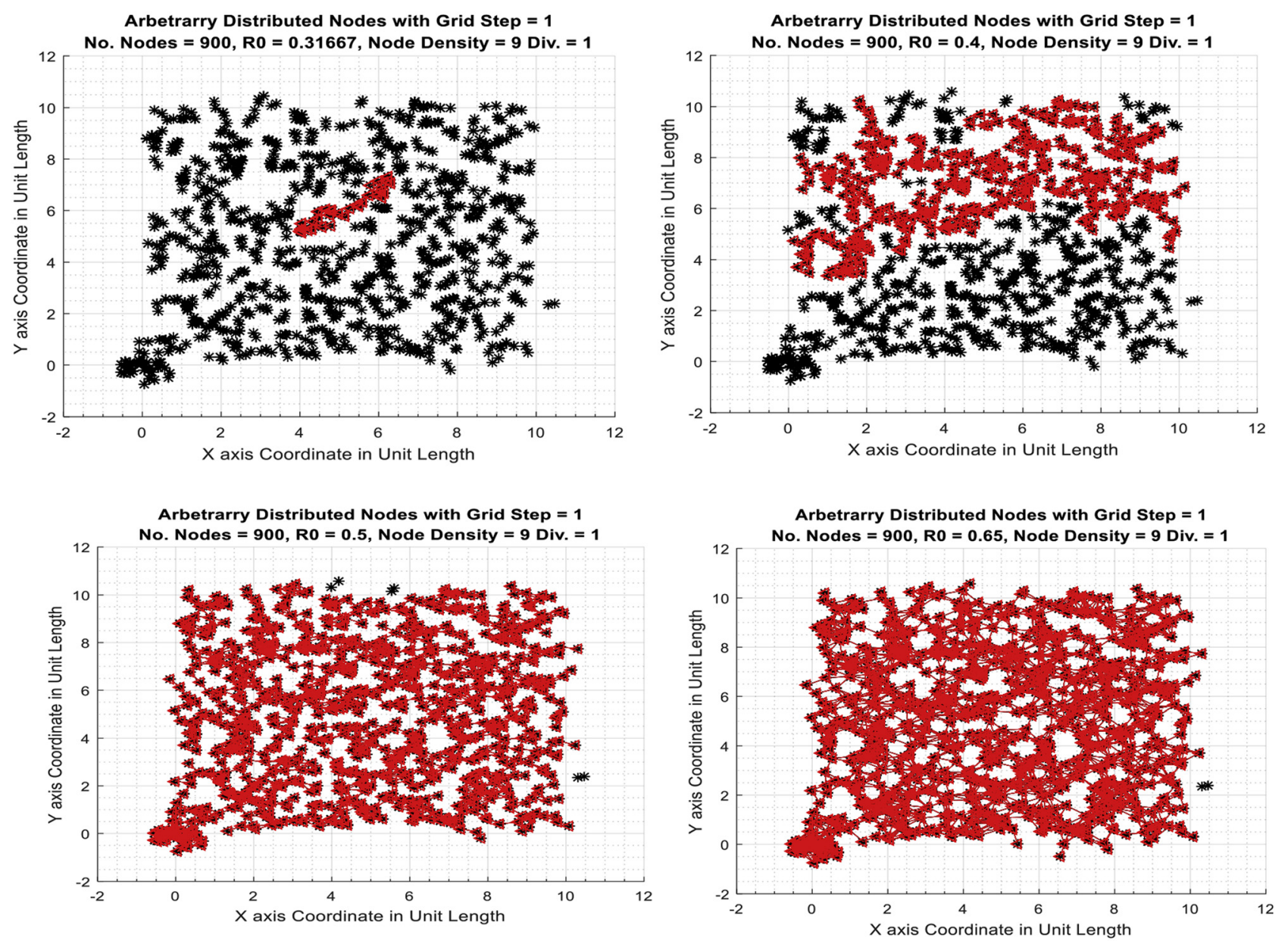

Fig. 8. Connected graph for $10 \times 10,9$ nodes/unit squared with $R_{0}=($ a) 0.31667 (b) 0.4 (c) 0.5 (d) 0.65 units 
is more logical to compare radii of inverse relation with the square root of depletion density to each other:

$$
R_{\max }=\frac{R_{0}}{\sqrt{D n}}
$$

Where $\mathrm{R}_{0}$ is maximum transmission radius for depletion density $D n=1$ and $R_{\max }$ is equivalent maximum transmission radius for larger densities.

Basic $R_{\max }$ and related radii for different densities obeying relation 7 are shown in Table 1 .

Network with 4 nodes per unit squared density and different maximum transmitted radios are plotted as shown in Fig. 7.

Network with 9 nodes per unit squared density and different maximum transmitted radios are also plotted as shown in Fig. 8.

The Longest connected chain in both densities in graphs show the same behavior as in lower node density where the connections are dominant for maximum radius of transmission greater than grid spacing divided by square root of node density as in Eq. (7). Then scaling of node densities can be confirmed for the same area of depletion.

Also, no significant signs are found and the minor differences in graph due to deviations may also be scaled. Number of nodes connected are scaled, while average connections per node about the same values as shown in Figs 9 and 10 .

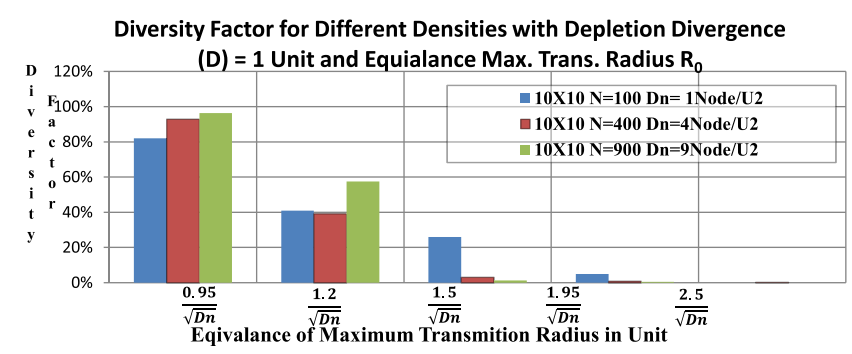

Fig. 9. Diversity factor for different depletion densities

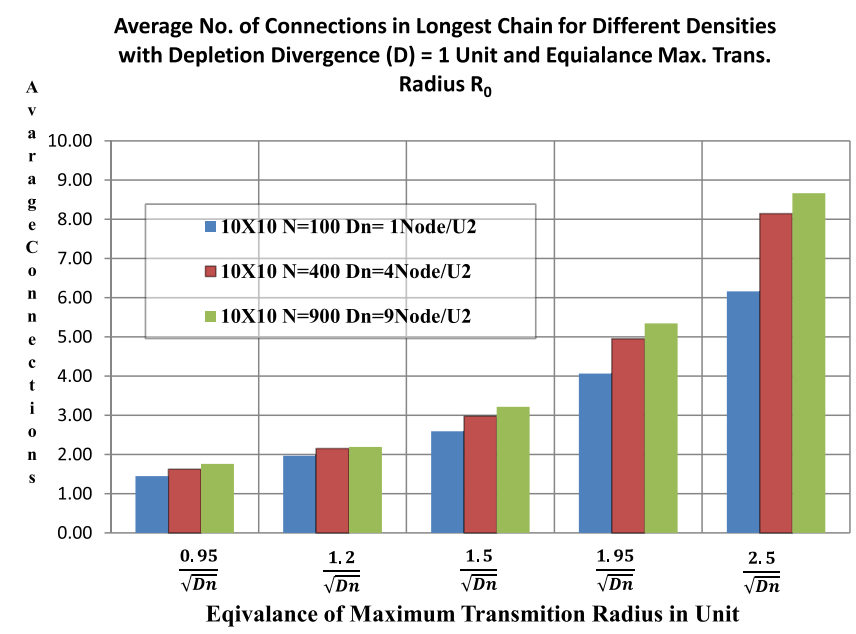

Fig. 10. Average No. of Connections for different depletion densities

\section{CONCLUSIONS AND FUTURE WORKS}

Wireless Sensor Networks are still a hot topic especially in mobile network and because it is part of more sophisticated articles dealing with the Internet of thing (IoT), (FOG) and CLOUD systems and technologies, since multiple objects must be connected with each other, and these relations must be geometrical, routing and smart connections. So that studding wireless sensor network as theoretical and geometrical properties will always support manufacturing and applying WSN in real word. Estimating the longest chain in connected network as a simple geometric configuration is one of many in this trained to study behavior and efficiency of network therefore to control, optimize network and so on. Knowledge of node coordinates in random depletion method is a partly solved problem and needs to be more researched in the future. The project simulates network as a simple disk model, while almost all WSNs are clustering configurations and it is important to upgrade model to deal with clustering configuration by using different maximum transmission radii for different nodes. Using different network models can be done in future works and may include elliptical or multi-hop transmission patterns.

No real efforts are found for finding longest node connected chain as a target method to compare, this trained of searching need to consider especially for data analysis and pattern recognition, this paper tries to give chain properties of nodes in a network more attentions.

Random distributed method in use is deviate $X$ and $Y$ coordinates from uniform grid with predefined step length, using different depletion methods can be helpful for more realistic presentations.

Find solutions to connect discriminated chains by inserting, mobile few nodes or increasing maximum transition range of specific nearest nodes between disconnected chains, these solutions may the topic of future projects.

\section{ACKNOWLEDGMENT}

The authors thank Mustansiriya University for its full support of this work. It is one of the Iraqi public universities named after the Mustansiriya school, which was founded in the time of the Abbasids in Baghdad in 1233 by the Caliph Al-Mustansir Billah. It was an important scientific and cultural center. Located in the capital Baghdad. Includes 13 faculties. Founded in 1963. www.uomustansiriyah.edu.iq.

\section{REFERENCES}

[1] A Survey on Centralized and Distributed Clustering Routing Algorithms for WSNs (PDF). IEEE 81st Vehicular Technology Conference, Glasgow, Scotland: IEEE Spring, 2015. https://doi.org/ 10.1109/VTCSpring.2015.7145650.2016. 
[2] I. F. Akyildiz and I.H. Kasimoglu, "Wireless Sensor and Actor Networks: Research Challenges," Ad Hoc Net. vol. 2, no. 4, pp. 351-367, 2004. https://doi.org/10.1016/j.adhoc.2004.04.003.

[3] W. Dargie and C. Poellabauer, Fundamentals of Wireless Sensor Networks: Theory and Practice, John Wiley and Sons, 2010, pp. 168-183. 191-192. https://doi.org/978-0-470-99765-9.

[4] K. Sohraby, D. Minoli, T. Znati, Wireless Sensor Networks: Technology, Protocols, and Applications, John Wiley and Sons, 2007, pp. 203-209. https://doi.org/978-0-471-74300-2.

[5] L. Sharma, "'Connectivity and Coverage Preserving Schemes for Surveillance Applications in WSN", School of Computer Science, Jaypee University of Informational Technology," Int. J. Comp. Appl. (0975 - 8887) vol. 50, no. 19, July 2012.

[6] R. Ali, "Abstract Performance \& Simulation Analysis of Sensor Area Coverage" MS in Computer Science, University of Nebraska, pp. 15-25, 2013.

[7] X. Xing, G. Wang, J. Wu, and J. Li, Square Region-Based Coverage and Connectivity Probability Model in Wireless Sensor Networks, 5th European Alliance for Innovation (EAI) International Conference on Collaborative Computing, Washington D.C., United States, 2009.

[8] P. Sameera, Using Local Geometry for Tunable Topology Control in Sensor Networks, Los Angeles, CA 90089, USA: Department of Electrical Engineering, University of Southern California, 2008.

[9] Y. Wang, X. -Y. Li. Localized Construction of Bounded Degree and Planar Spanner for Wireless Ad Hoc networks, San Diego, California, USA: Dept. of Computer Science Illinois Institute of Technology, DIALM-POMC'03, September 19, 2003. wangyu1@iit.edu.

[10] A. Tiwari, "Energy-efficient wireless sensor network design and implementation for condition-based maintenance," ACM Trans. Sensor Net. (TOSN), vol. 3, p. 1, 2007.

[11] B. Liu and D. Towsley. A Study of the Coverage of Large-Scale Sensor Networks. (2004). Computer Science Department Faculty Publication Series. 58. Retrieved from https://scholarworks.umass. edu/cs_faculty_pubs/58.

[12] M. Franceschetti and R. Meester, Random Networks for Communication: From Statistical Physics to Information Systems, 24. Cambridge University Press, 2007.

[13] T. Robles, “'Mobile wireless sensor networks: Modeling and anal$y$ sis of three-dimensional scenarios and neighbor discovery in mobile data collection". Ciudad Universitaria, Mexico," Ad Hoc Sensor Wireless Net. vol. 35, pp. 67-104, 2017.

[14] B. Wang, "Coverage problems in sensor networks: A survey," ACM Comput. Surv. vol. 43, no. 4, Article 32, October 2011. https://doi.org/10.1145/1978802.1978811.

[15] S. V. Ramanan and N. Kumareshan, "'Enhancing the life time of wireless sensor network by using the minimum energy scheduling algorithms". Department of Electronics and Communication Engineering Institute of Technology, Coimbatore - 35, India," Int. J. Innov. Res. Sci Eng. Technol. An ISO 3297: 2007 Certified Organization, vol. 3, Special Issue 1, February 2014. International Conference on Engineering Technology and Science.

[16] M. Bhardwaj, S. Misra, and G. Xue "Distributed Topology Control in Wireless Ad Hoc Networks using $\beta$-Skeletons". Department of Computer Science/College of Arts and Sciences/New Mexico State UniversityOffice: Science Hall 148- Phone: (575) 646-6256 Email: misra@cs.nmsu.edu.

[17] H. A. Alabbasi and A. H. Altimimy, "An approach to calculate exact coverage area for connected wireless sensor Network," J. Univ. Babylon. vol. 26, no. 7. Iraq, 2018.

[18] L. Liu, F. Xi, Z. Wang, J. Chen, and Y. Sun, Deployment Issues in Wireless Sensor Networks, (๑) Springer-Verlag Berlin Heidelberg, 2005.

[19] P. Gupta and P. R. Kumar, "Critical power for asymptotic connectivity in wireless networks," in Stochastic Analysis, Control, Optimization and Applications: A Volume in Honor of W.H. Fleming, W. M. McEneany, G. Yin, and Q. Zhang, Eds., (Boston), Birkhauser, 1998, pp. 547-566.

[20] K. Mondal, Designing Localization Algorithms for Wireless Sensor Networks: A Geometric Approach. Thesis submitted in partial fulfillment of the requirements for the degree of Doctor of Philosophy to the Department of Mathematics Indian Institute of Technology Guwahati. Guwahati - 781039, India, December 2014.

[21] S. Shakkottai, R. Srikant, and N. Shroff, "Unreliable sensor grids: Coverage, connectivity and diameter," in Proc. IEEE Infocom, 2003.

[22] "MATLAB Programming Fundamentals". The MathWorks, Inc. 3 Apple Hill Drive Natick, MA 01760-2098. @ COPYRIGHT 19842016.

[23] "MATLAB Function Reference", The MathWorks, Inc. 3 Apple Hill Drive Natick, MA 01760-2098. () COPYRIGHT 1984-2016. 